\section{Robust stability of moving horizon estimation under bounded disturbances}

\author{
Luo Ji, James B. Rawlings, Wuhua Hu, Andrew Wynn, \\ and Moritz Diehl
}

\begin{abstract}
This note proposes a new form of nonlinear state estimator for which we can establish robust global asymptotic stability (RGAS) in the case of bounded disturbances. In this estimator, a max term is added to the usual sum of stage costs, and one additional assumption is made relating the initial state stage cost to the system's detectability condition. A simulation example is presented to illustrate the estimator's performance. Two open issues are presented: (i) the proof of estimator convergence for convergent disturbances and (ii) changing from full information estimation to moving horizon estimation (MHE), which has a smaller and more tractable online computational complexity.
\end{abstract}

Index Terms-Moving horizon estimation, nonlinear state estimation, constraints, bounded disturbances, incremental input/output to state stability, robust global asymptotic stability.

\section{INTRODUCTION}

Moving horizon estimation (MHE) and its full information counterpart are online optimization-based state estimation methods that can handle nonlinear systems and satisfy constraints on the estimated states and disturbances. It has been established that the full information version can provide a robustly stable estimator in the case of convergent process and measurement disturbances [1, Theorem 12]. A remaining issue is whether the same property can be established for bounded (rather than convergent) disturbance [1, Conjecture 13]. There is some recent research on the issue, which assumes an alreadyknown deterministic estimator [2]. In this paper, we work on a more general case motivated by an early version of [3]. We show here that one can obtain a robustly stable full information estimator for bounded disturbances if one adds to the usual full information cost function a max stage cost term, and makes one additional assumption linking the chosen estimator stage cost to the detectability condition of the nonlinear system.

The paper is organized as follows. First we define notation and introduce the standard and modified state estimation cost functions. We then state the chosen definition for nonlinear detectability, and define robust global asymptotic stability (RGAS) of a state estimator. Then we prove the main result of the paper: the full information estimator with the modified cost function is RGAS for a detectable nonlinear system subject to bounded disturbances. A simulation example is then presented to compare the performances of the state estimators using the conventional and the modified cost functions.

\section{Preliminaries and Problem Description}

The symbols $\mathbb{I}_{\geq 0}$ and $\mathbb{R}_{\geq 0}$ denote the sets of nonnegative integers and reals, respectively. The symbol $\mathbb{I}_{0: N-1}$ denotes the set $\{0,1, \ldots, N-1\}$. The symbol $|\cdot|$ denotes the Euclidean norm. The bold symbol $\mathbf{x}$, denote a sequence of a vector-valued variable $x$,

Manuscript received November 15, 2013. Revision submitted September 23, 2014.

L. Ji and J. B. Rawlings (IEEE Fellow) are with Department of Chemical and Biological Engineering, University of Wisconsin-Madison, Madison, WI USA (e-mail: 1ji2@wisc.edu, rawlings@engr.wisc.edu).

W. Hu is with the School of Electrical and Electronic Engineering, Nanyang Technological University, Singapore (e-mail: hwh@ntu.edu.sg).

Andrew Wynn is with Department of Aeronautics, Imperial College, London SW7 2AZ, United Kingdom (e-mail: a.wynn@imperial.ac.uk).

Moritz Diehl is with Department of Microsystems Engineering (IMTEK), University of Freiburg, Germany, and Department of Electrical Engineering (ESAT-STADIUS), KU Leuven University, Belgium.(e-mail: moritz.diehl@imtek.uni-freiburg.de). $\{x(0), x(1), \ldots\}$. The notation $\|\mathbf{x}\|$ is the sup norm over a sequence, $\sup _{i \geq 0}|x(i)|$, and $\|\mathbf{x}\|_{a: b}$ denotes $\max _{a \leq i \leq b}|x(i)|$. The definition of system detectability and statements and proofs of estimator stability are significantly streamlined using the definitions and properties of $\mathcal{K}$ and $\mathcal{K} \mathcal{L}$ functions, so we provide a brief summary here. The interested reader may also wish to consult [4, pp. 144-147] and [5, Appendix B] for further discussion.

Definition 1 ( $\mathcal{K}, \mathcal{K}_{\infty}$, and $\mathcal{K} \mathcal{L}$ functions). A function $\sigma: \mathbb{R}_{\geq 0} \rightarrow$ $\mathbb{R}_{\geq 0}$ belongs to class $\mathcal{K}$ if it is continuous, zero at zero, and strictly increasing; $\sigma: \mathbb{R}_{\geq 0} \rightarrow \mathbb{R}_{\geq 0}$ belongs to class $\mathcal{K}_{\infty}$ if it is a class $\mathcal{K}$ and unbounded $(\sigma(s) \rightarrow \infty$ as $s \rightarrow \infty)$. A function $\beta: \mathbb{R}_{>0} \times$ $\mathbb{I}_{\geq 0} \rightarrow \mathbb{R}_{\geq 0}$ belongs to class $\mathcal{K} \mathcal{L}$ if it is continuous and if, for each $t \in \mathbb{I}_{\geq 0}, \bar{\beta}(\cdot, t)$ is a class $\mathcal{K}$ function and for each $r \geq 0, \beta(r, \cdot)$ is nonincreasing and satisfies $\lim _{t \rightarrow \infty} \beta(r, t)=0$.

We require the following basic inequalities to streamline our presentation. Proofs of these properties are given in [1].

1) For $\gamma(\cdot) \in \mathcal{K}$, the following holds for all $a_{i} \in \mathbb{R}_{\geq 0}, i \in \mathbb{I}_{1: n}$

$$
\frac{1}{n} \sum_{i=1}^{n} \gamma\left(a_{i}\right) \leq \gamma\left(a_{1}+a_{2}+\cdots+a_{n}\right) \leq \sum_{i=1}^{n} \gamma\left(n a_{i}\right)
$$

2) Similarly, for $\beta(\cdot) \in \mathcal{K} \mathcal{L}$ the following holds for all $a_{i} \in \mathbb{R}_{\geq 0}$, $i \in \mathbb{I}_{1: n}$, and all $t \in \mathbb{R}_{\geq 0}$

$$
\frac{1}{n} \sum_{i=1}^{n} \beta\left(a_{i}, t\right) \leq \beta\left(\left(a_{1}+a_{2}+\cdots+a_{n}\right), t\right) \leq \sum_{i=1}^{n} \beta\left(n a_{i}, t\right)
$$

We assume that the system generating the measurements is given by the standard discrete time, nonlinear system

$$
x^{+}=f(x, w) \quad y=h(x)+v
$$

The state of the system is $x \in \mathbb{R}^{n}$, the measurement is $y \in \mathbb{R}^{p}$, and the notation $x^{+}$means $x$ at the next sample time. A control input $u$ may be included in the model, but it is considered a known variable, and its inclusion is irrelevant to state estimation, so we suppress it in the model under consideration here. We assume throughout that functions $f: \mathbb{R}^{n} \times \mathbb{R}^{g} \rightarrow \mathbb{R}^{n}$ and $h: \mathbb{R}^{n} \rightarrow \mathbb{R}^{p}$ are continuous.

We receive a measurement $y$ from the sensor, but the process disturbance, $w \in \mathbb{R}^{g}$, measurement disturbance, $v \in \mathbb{R}^{p}$, and system initial state, $x(0)$, are considered unknown variables. We model $w, v, x(0)$ as unknown, but bounded disturbance variables.

Definition 2 (Bounded sequences; set $\mathbb{B}$ ). A sequence $w(k), k \geq 0$ is bounded if $\|\mathbf{w}\|$ is bounded. The set of bounded sequences is denoted by $\mathbb{B}$.

\section{A. Full information estimation}

First we define some notation necessary to distinguish the system variables from the estimator variables. We have already introduced the system variables $(x, w, v)$ in (3). In the estimator optimization problem, these have corresponding decision variables, which we denote $(\chi, \omega, \nu)$. The optimal decision variables are denoted $(\hat{x}, \hat{w}, \hat{v})$ and these optimal decisions are the estimates provided by the state estimator. The relationships between these variables are

$$
\begin{array}{ll}
\chi^{+}=f(\chi, \omega) & y=h(\chi)+\nu \\
\hat{x}^{+}=f(\hat{x}, \hat{w}) & y=h(\hat{x})+\hat{v}
\end{array}
$$

Notice that it is always the system measurement $y$ that appears in the second column of equations.

We begin with a reasonably general definition of the full information estimator that produces an estimator that is stable. The full 
information objective function is traditionally defined for current time $T \geq 1$ as

$$
V_{T}^{\mathrm{sum}}(\chi(0), \boldsymbol{\omega})=\ell_{x}\left(\chi(0)-\bar{x}_{0}\right)+\sum_{i=0}^{T-1} \ell_{i}(\omega(i), \nu(i))
$$

subject to (4) in which $y(i)$ is the measurement at time $i$, and $\bar{x}_{0}$ is a user-defined prior value of the initial state. Because $\nu=y-h(\chi)$ is the error in fitting the measurement $y, \ell_{i}(\omega, \nu)$ costs the model disturbance and the fitting error. These are the two error sources that we reconcile in all state estimation problems.

Next we define a modified objective function, such that we can establish stability properties under bounded disturbances rather than convergent disturbances

$$
\begin{aligned}
& V_{T}(\chi(0), \boldsymbol{\omega})=\frac{1}{T}(1+\delta) \ell_{x}\left(\chi(0)-\bar{x}_{0}\right)+ \\
& \frac{1}{T} \sum_{i=0}^{T-1} \ell_{i}(\omega(i), \nu(i))+\delta \max _{i \in 0: T-1} \ell_{i}(\omega(i), \nu(i))
\end{aligned}
$$

subject to (4) in which $\delta$ is a scalar weighting parameter chosen by the user. This modified objective could be viewed as the conventional objective plus a weighted maximum of $\ell_{i}$; note that when $\delta=0$, the estimator is equivalent to the standard estimation given in (6), because for a specific $T$, it does not change the optimization result of (6) if we divide $V_{T}^{\text {sum }}$ by $T$. On the other hand, we could suppress the sum of $\ell_{i}$ term by letting $\delta \rightarrow \infty$

$$
V_{T}^{\max }(\chi(0), \boldsymbol{\omega})=\frac{1}{T} \ell_{x}\left(\chi(0)-\bar{x}_{0}\right)+\max _{i \in 0: T-1} \ell_{i}(\omega(i), \nu(i))
$$

subject to (4).

Definition 3 (Full information estimation). The full information estimator at time $T$ is the solution to

$$
\text { FI : } \quad V_{T}^{0}:=\min _{\chi(0), \boldsymbol{\omega}} V_{T}(\chi(0), \boldsymbol{\omega}) \quad \text { subject to: (4) }
$$

The optimal decisions are denoted $\hat{x}(0 \mid T)$ and $\widehat{\mathbf{w}}$. Notice that the optimal decisions depend on the measurements $y(i), i=0,1, \ldots, T$, but we supress this dependence for more compact notation. Using the optimal solution and (5), we can solve for all $\hat{x}(i \mid T), i=0, \cdots, T$, which is the (smoothed) estimated trajectory. For each time $T$ we take the last result $\hat{x}(T \mid T)$ as the current state estimate, which is the usual estimate passed to a controller. Estimate error is then defined for $k \geq 0$ as the difference between the system state generating the data and the optimal estimate based on data up to time $k$

$$
\tilde{x}(k)=x(k)-\hat{x}(k \mid k)
$$

We also consider full information using $V_{T}^{\text {sum }}$ or $V_{T}^{\max }$ instead of $V_{T}$. For simplicity, we call these the (full information) MIX, SUM, and MAX estimators when using objectives (7), (6), and (8), respectively.

Assumption 4 (Positive definite stage cost). The stage costs are continuous functions and satisfy the following inequalities for all $w \in \mathbb{R}^{g}$, and $v \in \mathbb{R}^{p}$

$$
\begin{aligned}
\underline{\gamma}_{x}(|x|) & \leq \ell_{x}(x) \leq \bar{\gamma}_{x}(|x|) \\
\underline{\gamma}_{w}(|w|)+\underline{\gamma}_{v}(|v|) & \leq \ell_{i}(w, v) \leq \bar{\gamma}_{w}(|w|)+\bar{\gamma}_{v}(|v|)
\end{aligned}
$$

in which $\underline{\gamma}_{x}, \bar{\gamma}_{x}, \underline{\gamma}_{w}, \bar{\gamma}_{w}, \underline{\gamma}_{v}, \bar{\gamma}_{v} \in \mathcal{K}_{\infty}$.

Remark 5. From Assumption 4 the following holds for all $\mathbf{w}, \mathbf{v} \in \mathbb{B}$

$$
\sup _{i \geq 0} \ell_{i}(w(i), v(i)) \leq \bar{\gamma}_{w}(\|\mathbf{w}\|)+\bar{\gamma}_{v}(\|\mathbf{v}\|)
$$

Remark 6. The two objectives satisfy for all $T \geq 1, \chi(0) \in \mathbb{R}^{n}$, $\boldsymbol{\omega} \in \mathbb{R}^{T g}$,

$\frac{1}{T}(1+\delta) V_{T}^{\text {sum }}(\chi(0), \boldsymbol{\omega}) \leq V_{T}(\chi(0), \boldsymbol{\omega}) \leq(1+\delta) V_{T}^{\text {sum }}(\chi(0), \boldsymbol{\omega})$

Remark 7. Note that the MIX, SUM, and MAX estimators are well defined because the optimal solutions to each of these estimators exists. The solution to the SUM estimator exists for all $T \geq 1$ because (1) the cost $V_{T}(\cdot)$ is continuous due to the continuity of $f(\cdot)$ and $h(\cdot)$, and (2) $V_{T}(\cdot)$ is radially unbounded in the decision variables due to the lower bounds in (11), (12) of Assumption 4. Continuity plus radial unboundedness implies existence of the optimal solution by the Weierstrass theorem. Note the same existence argument can be made for the MIX and MAX estimators after we transform their objectives into their smoothed versions, as shown in Section IV-B.

We take incremental input/output-to-state stability (i-IOSS) as the definition of detectability for nonlinear systems [6].

Definition 8 (i-IOSS). The system $x^{+}=f(x, w), y=h(x)$ is incrementally input/output-to-state stable (i-IOSS) if there exist functions $\alpha(\cdot, \cdot) \in \mathcal{K} \mathcal{L}$ and $\gamma_{1}(\cdot), \gamma_{2}(\cdot) \in \mathcal{K}$ such that for every two initial states $z_{1}$ and $z_{2}$, and any two disturbance sequences $\mathbf{w}_{1}$ and $\mathbf{w}_{2}$ generating state sequences $\mathbf{x}_{1}\left(z_{1}, \mathbf{w}_{1}\right)$ and $\mathbf{x}_{2}\left(z_{2}, \mathbf{w}_{2}\right)$, the following holds for all $k \geq 1$

$$
\begin{aligned}
& \left|x\left(k ; z_{1}, \mathbf{w}_{1}\right)-x\left(k ; z_{2}, \mathbf{w}_{2}\right)\right| \leq \alpha\left(\left|z_{1}-z_{2}\right|, k\right)+ \\
& \quad \gamma_{1}\left(\left\|\mathbf{w}_{1}-\mathbf{w}_{2}\right\|_{0: k-1}\right)+\gamma_{2}\left(\left\|h\left(\mathbf{x}_{1}\right)-h\left(\mathbf{x}_{2}\right)\right\|_{0: k-1}\right)
\end{aligned}
$$

The notation $x\left(k ; x_{0}, \mathbf{w}\right)$ denotes the solution to $x^{+}=f(x, w)$ satisfying initial condition $x(0)=x_{0}$ with disturbance sequence $\mathbf{w}=\{w(0), w(1), \ldots\}$. The notation $h(\mathbf{x})$ is then defined as $\left\{h\left(x\left(0 ; x_{0}, \mathbf{w}\right)\right), h\left(x\left(1 ; x_{0}, \mathbf{w}\right)\right), \ldots\right\}$. The notation $x(k ; \hat{x}(0 \mid k), \widehat{\mathbf{w}})$ therefore denotes the optimal estimate at time $k$ based on data up to time $k$.

Definition 9 (Robust global asymptotic stability (RGAS)). The estimate is based on the noisy measurement $\mathbf{y}=h\left(\mathbf{x}\left(x_{0}, \mathbf{w}\right)\right)+\mathbf{v}$. The estimate is RGAS if for all $x_{0}$ and $\bar{x}_{0}$, and bounded $(\mathbf{w}, \mathbf{v})$, there exists functions $\phi(\cdot, \cdot) \in \mathcal{K} \mathcal{L}$ and $\pi_{w}(\cdot), \pi_{v}(\cdot) \in \mathcal{K}$ such that the following holds for all $k \geq 1$

$$
\begin{aligned}
\left|x\left(k ; x_{0}, \mathbf{w}\right)-x(k ; \hat{x}(0 \mid k), \widehat{\mathbf{w}})\right| \leq \phi\left(\left|x_{0}-\bar{x}_{0}\right|, k\right) \\
+\pi_{w}\left(\|\mathbf{w}\|_{0: k-1}\right)+\pi_{v}\left(\|\mathbf{v}\|_{0: k-1}\right)
\end{aligned}
$$

Remark 10. The main characteristic of the RGAS definition is that the dynamic system generating the estimate error is input-to-state stable (ISS) [7] considering $(w, v)$ as the input.

Finally we make an additional assumption that enables us to establish the later properties.

Assumption 11 (Separability of i-IOSS $\mathcal{K} \mathcal{L}$ and $\mathcal{K}$ functions). The $\mathcal{K} \mathcal{L}$ function $\alpha(r, k)$ in Definition 8 satisfies the following separability condition. There exist positive scalars $c_{\alpha}, p, a>0$ such that $\alpha(r, k) \leq c_{\alpha} r^{p} k^{-a}$ holds for all $r \geq 0, k \geq 1$

Assumption 12 (Choices of stage costs). The initial state stage cost $\ell_{x}(\cdot)$ is chosen so that its lower and upper bounds satisfy $\underline{\gamma}_{x}(r)=$ $\underline{c}_{x} r^{q}$ for some $\underline{c}_{x}>0$ and $q>p / a$.

These assumptions strengthen the detectability condition and link the initial state stage cost to the detectability condition through the condition $q>p / a$. See [3] for some generalizations of this assumption. We next show that this requirement is sufficient to establish RGAS of the MIX estimator. 


\section{MAIN RESULT}

We can now state and prove the main result of the paper.

Theorem 13 (RGAS of full information estimate for bounded disturbances). Consider an i-IOSS (detectable) system satisfying Assumptions 11 and 12 with measurement sequence generated by (3), bounded disturbances satisfying Definition 2, and stage cost satisfying Assumption 4. Then the full information (MIX) estimator is $R G A S$.

Proof: First from i-IOSS (13), we have the upper bound of the estimation error

$$
\begin{aligned}
\mid x\left(k ; x_{0}, \mathbf{w}\right)- & x(k ; \hat{x}(0 \mid k), \widehat{\mathbf{w}}) \mid \leq \alpha\left(\left|x_{0}-\hat{x}(0 \mid k)\right|, k\right)+ \\
& \gamma_{1}\left(\|\mathbf{w}-\widehat{\mathbf{w}}\|_{0: k-1}\right)+\gamma_{2}\left(\|\mathbf{v}-\widehat{\mathbf{v}}\|_{0: k-1}\right)
\end{aligned}
$$

For each $k \geq 1$, the optimal MIX objective function can be expressed as

$$
\begin{aligned}
V_{k}^{0}:= & \left.V_{k}(\hat{x}(0 \mid k)), \widehat{\mathbf{w}}_{k}\right)=\frac{1}{k}(1+\delta) \ell_{x}\left(\hat{x}(0 \mid k)-\bar{x}_{0}\right)+ \\
& \frac{1}{k} \sum_{i=0}^{k-1} \ell_{i}(\hat{w}(i \mid k), \hat{v}(i \mid k))+\delta \max _{i \in 0: k-1} \ell_{i}(\hat{w}(i \mid k), \hat{v}(i \mid k))
\end{aligned}
$$

From optimality we know that

$$
\begin{aligned}
V_{k}^{0} \leq V_{k}\left(x_{0}, \mathbf{w}\right) & =\frac{1}{k}(1+\delta) \ell_{x}\left(x_{0}-\bar{x}_{0}\right)+ \\
& \frac{1}{k} \sum_{i=0}^{k-1} \ell_{i}(w(i), v(i))+\delta \max _{i \in 0: k-1} \ell_{i}(w(i), v(i))
\end{aligned}
$$

Then from Remark 6, Assumption 4 and Remark 5 we have the following upper bound $\bar{V}_{k}$ of $V_{k}^{0}$ valid for $k \geq 1$

$$
\begin{aligned}
& V_{k}^{0} \leq \bar{V}_{k}:=(1+\delta)\left(\frac{1}{k} \bar{\gamma}_{x}\left(\left|x_{0}-\bar{x}_{0}\right|\right)+\right. \\
&\left.\bar{\gamma}_{w}(\|\mathbf{w}\|)+\bar{\gamma}_{v}(\|\mathbf{v}\|)\right)
\end{aligned}
$$

Also from Assumption 4 we have the following lower bound of $V_{k}^{0}$

$$
\begin{aligned}
& V_{k}^{0} \geq \frac{1}{k}(1+\delta) \underline{\gamma}_{x}\left(\left|\hat{x}(0 \mid k)-\bar{x}_{0}\right|\right)+ \\
& \delta \underline{\gamma}_{w}(|\hat{w}(i \mid k)|)+\delta \underline{\gamma}_{v}(|\hat{v}(i \mid k)|)
\end{aligned}
$$

for any time $i \leq k-1$. Next we proceed to establish an upper bound for $\left\|\mathbf{w}-\widehat{\mathbf{w}}_{k}\right\|_{0: k-1}$. From the triangle inequality and definition of the sup norm we have that

$$
\left\|\mathbf{w}-\widehat{\mathbf{w}}_{k}\right\|_{0: k-1} \leq\|\mathbf{w}\|_{0: k-1}+\left\|\widehat{\mathbf{w}}_{k}\right\|_{0: k-1}
$$

Next we require a bound for $\left\|\widehat{\mathbf{w}}_{k}\right\|_{0: k-1}$. We have from (17) and (16) that $\underline{\gamma}_{w}(|\hat{w}(i \mid k)|) \leq(1 / \delta) \bar{V}_{k}$ for all $i \leq k-1$. This implies that $\left\|\widehat{\mathbf{w}}_{k}\right\|_{0: k-1} \leq \underline{\gamma}_{w}^{-1}\left((1 / \delta) \bar{V}_{k}\right)$, and substituting (16) into this result and using (1) gives

$$
\begin{aligned}
\left\|\widehat{\mathbf{w}}_{k}\right\|_{0: k-1} & \leq \underline{\gamma}_{w}^{-1}\left(\frac{1+\delta}{\delta} \frac{3}{k} \bar{\gamma}_{x}\left(\left|x_{0}-\bar{x}_{0}\right|\right)\right)+ \\
& +\underline{\gamma}_{w}^{-1}\left(\frac{1+\delta}{\delta} 3 \bar{\gamma}_{w}(\|\mathbf{w}\|)\right)+\underline{\gamma}_{w}^{-1}\left(\frac{1+\delta}{\delta} 3 \bar{\gamma}_{v}(\|\mathbf{v}\|)\right)
\end{aligned}
$$

Applying $\gamma_{1}(\cdot)$ to (18) and using (19) gives

$$
\begin{aligned}
\gamma_{1} & \left(\left\|\mathbf{w}-\widehat{\mathbf{w}}_{k}\right\|_{0: k-1}\right) \leq \gamma_{1}\left(\|\mathbf{w}\|+\left\|\widehat{\mathbf{w}}_{k}\right\|\right) \\
\leq & \gamma_{1}\left(\|\mathbf{w}\|+\underline{\gamma}_{w}^{-1}\left(\frac{1+\delta}{\delta} \frac{3}{k} \bar{\gamma}_{x}\left(\left|x_{0}-\bar{x}_{0}\right|\right)\right)\right. \\
& \left.+\underline{\gamma}_{w}^{-1}\left(\frac{1+\delta}{\delta} 3 \bar{\gamma}_{w}(\|\mathbf{w}\|)\right)+\underline{\gamma}_{w}^{-1}\left(\frac{1+\delta}{\delta} 3 \bar{\gamma}_{v}(\|\mathbf{v}\|)\right)\right) \\
\leq & \gamma_{1}\left(3 \underline{\gamma}_{w}^{-1}\left(\frac{1+\delta}{\delta} \frac{3}{k} \bar{\gamma}_{x}\left(\left|x_{0}-\bar{x}_{0}\right|\right)\right)\right) \\
& +\gamma_{1}\left(3\|\mathbf{w}\|+3 \underline{\gamma}_{w}^{-1}\left(\frac{1+\delta}{\delta} 3 \bar{\gamma}_{w}(\|\mathbf{w}\|)\right)\right) \\
& +\gamma_{1}\left(3 \underline{\gamma}_{w}^{-1}\left(\frac{1+\delta}{\delta} 3 \bar{\gamma}_{v}(\|\mathbf{v}\|)\right)\right)
\end{aligned}
$$

Noting that $\beta(r, k):=(1 / k) \bar{\gamma}_{x}(r)$ is $\mathcal{K} \mathcal{L}$, and using the properties of $\mathcal{K}$ and $\mathcal{K} \mathcal{L}$ functions, this equation can be expressed as

$$
\begin{aligned}
\gamma_{1}\left(\left\|\mathbf{w}-\widehat{\mathbf{w}}_{k}\right\|_{0: k-1}\right) \leq \phi_{x}^{w}\left(\left|x_{0}-\bar{x}_{0}\right|, k\right)+ & \\
& \pi_{w}^{w}(\|\mathbf{w}\|)+\pi_{v}^{w}(\|\mathbf{v}\|)
\end{aligned}
$$

with $\phi_{x}^{w} \in \mathcal{K} \mathcal{L}$ and $\pi_{w}^{w}, \pi_{v}^{w} \in \mathcal{K}$. Notice that the same reasoning applies to $\left\|\mathbf{v}-\widehat{\mathbf{v}}_{k}\right\|_{0: k-1}$ yielding

$$
\begin{aligned}
\gamma_{2}\left(\left\|\mathbf{v}-\widehat{\mathbf{v}}_{k}\right\|_{0: k-1}\right) \leq \phi_{x}^{v}\left(\left|x_{0}-\bar{x}_{0}\right|, k\right)+ \\
\pi_{w}^{v}(\|\mathbf{w}\|)+\pi_{v}^{v}(\|\mathbf{v}\|)
\end{aligned}
$$

for $\phi_{x}^{v} \in \mathcal{K} \mathcal{L}$ and $\pi_{w}^{v}, \pi_{v}^{v} \in \mathcal{K}$.

From (17) and (16) we also have that

$$
\begin{aligned}
\underline{\gamma}_{x}\left(\left|\hat{x}(0 \mid k)-\bar{x}_{0}\right|\right) & \leq \frac{k}{1+\delta} V_{k}^{0} \leq \frac{k}{1+\delta} \bar{V}_{k} \leq \\
& \bar{\gamma}_{x}\left(\left|x_{0}-\bar{x}_{0}\right|\right)+k \bar{\gamma}_{w}(\|\mathbf{w}\|)+k \bar{\gamma}_{v}(\|\mathbf{v}\|)
\end{aligned}
$$

Then taking the inverse of the $\mathcal{K}$ function and using (1) generates

$$
\begin{aligned}
\left|\hat{x}(0 \mid k)-\bar{x}_{0}\right| \leq & \underline{\gamma}_{x}^{-1}\left(\bar{\gamma}_{x}\left(\left|x_{0}-\bar{x}_{0}\right|\right)\right. \\
& \left.+k \bar{\gamma}_{w}(\|\mathbf{w}\|)+k \bar{\gamma}_{v}(\|\mathbf{v}\|)\right) \\
\leq & \underline{\gamma}_{x}^{-1}\left(3 \bar{\gamma}_{x}\left(\left|x_{0}-\bar{x}_{0}\right|\right)\right) \\
& +\underline{\gamma}_{x}^{-1}\left(3 k \bar{\gamma}_{w}(\|\mathbf{w}\|)\right)+\underline{\gamma}_{x}^{-1}\left(3 k \bar{\gamma}_{v}(\|\mathbf{v}\|)\right)
\end{aligned}
$$

Again from the triangle inequality we have that

$$
\begin{aligned}
& \left|\hat{x}(0 \mid k)-x_{0}\right|=\left|\left(\hat{x}(0 \mid k)-\bar{x}_{0}\right)-\left(x_{0}-\bar{x}_{0}\right)\right| \\
& \leq\left|\hat{x}(0 \mid k)-\bar{x}_{0}\right|+\left|x_{0}-\bar{x}_{0}\right| \\
& \leq\left|x_{0}-\bar{x}_{0}\right|+\underline{\gamma}_{x}^{-1}\left(3 \bar{\gamma}_{x}\left(\left|x_{0}-\bar{x}_{0}\right|\right)\right) \\
& \quad+\underline{\gamma}_{x}^{-1}\left(3 k \bar{\gamma}_{w}(\|\mathbf{w}\|)\right)+\underline{\gamma}_{x}^{-1}\left(3 k \bar{\gamma}_{v}(\|\mathbf{v}\|)\right)
\end{aligned}
$$

Then we have the upper bound of the first part of (15) as

$$
\begin{aligned}
& \alpha\left(\left|\hat{x}(0 \mid k)-x_{0}\right|, k\right) \\
& \leq \alpha\left(\left|x_{0}-\bar{x}_{0}\right|+\underline{\gamma}_{x}^{-1}\left(3 \bar{\gamma}_{x}\left(\left|x_{0}-\bar{x}_{0}\right|\right)\right)\right. \\
& \left.\quad+\underline{\gamma}_{x}^{-1}\left(3 k \bar{\gamma}_{w}(\|\mathbf{w}\|)\right)+\underline{\gamma}_{x}^{-1}\left(3 k \bar{\gamma}_{v}(\|\mathbf{v}\|)\right), k\right) \\
& \leq \alpha\left(3\left|x_{0}-\bar{x}_{0}\right|+3 \underline{\gamma}_{x}^{-1}\left(3 \bar{\gamma}_{x}\left(\left|x_{0}-\bar{x}_{0}\right|\right)\right), k\right) \\
& \quad+\alpha\left(3 \underline{\gamma}_{x}^{-1}\left(3 k \bar{\gamma}_{w}(\|\mathbf{w}\|)\right), k\right)+\alpha\left(3 \underline{\gamma}_{x}^{-1}\left(3 k \bar{\gamma}_{v}(\|\mathbf{v}\|)\right), k\right)
\end{aligned}
$$

The first term on the right-hand side of the inequality is a $\mathcal{K} \mathcal{L}$ function. Using Assumption 11, the second term satisfies

$$
\begin{aligned}
& \alpha\left(3 \underline{\gamma}_{x}^{-1}\left(3 k \bar{\gamma}_{w}(\|\mathbf{w}\|)\right), k\right) \leq \\
& \quad\left(\frac{c_{\alpha} 3^{(p+p / q)}}{\underline{c}_{x}^{p / q}}\right) k^{p / q-a} \bar{\gamma}_{w}(\|\mathbf{w}\|)^{p / q}:=\phi_{w}^{x}(\|\mathbf{w}\|, k)
\end{aligned}
$$


Note that due to Assumption 11, $p / q-a<0$ and $\phi_{w}^{x}$ is therefore a $\mathcal{K} \mathcal{L}$ function. Similar analysis applies to the third term giving

$$
\begin{aligned}
& \alpha\left(\left|\hat{x}(0 \mid k)-x_{0}\right|, k\right) \leq \phi_{x}^{x}\left(\left|x_{0}-\bar{x}_{0}\right|, k\right)+ \\
& \phi_{w}^{x}(\|\mathbf{w}\|, k)+\phi_{v}^{x}(\|\mathbf{v}\|, k)
\end{aligned}
$$

for $\phi_{x}^{x}, \phi_{w}^{x}, \phi_{v}^{x} \in \mathcal{K} \mathcal{L}$. Note that $\phi_{w}^{x}(\|\mathbf{w}\|, k) \leq \phi_{w}^{x}(\|\mathbf{w}\|, 1)$ and $\phi_{v}^{x}(\|\mathbf{v}\|, k) \leq \phi_{v}^{x}(\|\mathbf{v}\|, 1)$ giving

$$
\begin{aligned}
\alpha\left(\left|\hat{x}(0 \mid k)-x_{0}\right|, k\right) \leq \phi_{x}^{x}\left(\left|x_{0}-\bar{x}_{0}\right|, k\right)+ \\
\pi_{w}^{x}(\|\mathbf{w}\|)+\pi_{v}^{x}(\|\mathbf{v}\|)
\end{aligned}
$$

for $\pi_{w}^{x}, \pi_{v}^{x} \in \mathcal{K}$. We substitute (22), (21), and (20) into (15) to obtain for all $k \geq 1$,

$$
\begin{aligned}
& \left|x\left(k ; x_{0}, \mathbf{w}\right)-x\left(k ; \hat{x}(0 \mid k), \widehat{\mathbf{w}}_{k}\right)\right| \leq \\
& \quad \phi\left(\left|x_{0}-\bar{x}_{0}\right|, k\right)+\pi_{w}(\|\mathbf{w}\|)+\pi_{v}(\|\mathbf{v}\|)
\end{aligned}
$$

in which $\phi:=\phi_{x}^{x}+\phi_{x}^{w}+\phi_{x}^{v} \in \mathcal{K} \mathcal{L}$, and $\pi_{w}:=\pi_{w}^{x}+\pi_{w}^{w}+\pi_{w}^{v} \in \mathcal{K}$, and $\pi_{v}:=\pi_{v}^{x}+\pi_{x}^{w}+\pi_{v}^{v} \in \mathcal{K}$. Since $w(j), v(j)$ for $j \geq k$ affect neither $x(k)$ nor $\hat{x}(k \mid k)$, this result also implies that

$$
\begin{aligned}
& \left|x\left(k ; x_{0}, \mathbf{w}\right)-x\left(k ; \hat{x}(0 \mid k), \widehat{\mathbf{w}}_{k}\right)\right| \leq \\
& \phi\left(\left|x_{0}-\bar{x}_{0}\right|, k\right)+\pi_{w}\left(\|\mathbf{w}\|_{0: k-1}\right)+\pi_{v}\left(\|\mathbf{v}\|_{0: k-1}\right)
\end{aligned}
$$

The estimate error therefore satisfies (14) and RGAS has been established.

Note that RGAS of the MAX estimator can be established similarly.

\section{DISCUSSION}

\section{A. Discussion of Assumptions 11 and 12}

The new restriction in this paper is Assumption 11, so we elaborate further on this assumption. If we step back, remove some details, and look at the general issue that is being addressed in Assumption 11, it is basically this. When can the linear growth in the first term of a $\mathcal{K} \mathcal{L}$ function be overcome by the decrease in its second argument, i.e., for what $\alpha(\cdot) \in \mathcal{K} \mathcal{L}$, does there exist $\gamma(\cdot) \in \mathcal{K}$ such that for some $\beta(\cdot)$ in $\mathcal{K} \mathcal{L}$

$$
\alpha(\gamma(r k), k) \leq \beta(r, k)
$$

for all $r \geq 0$, and $k \geq 1$ ? This general question appears to be rather new and unexplored, especially here because it arises in the context of the system's detectability, which is already a complex issue for nonlinear systems.

There are, however, some important cases in which Assumption 11 clearly is satisfied, and Assumption 12 is not required. The first case is nonlinear observability. Nonlinear observability rather than detectability corresponds to the case of $\alpha(\cdot)=0$ in Definition 8 .

Remark 14. Assumption 11 is satisfied for a nonlinear observable system (corresponding to $\alpha(\cdot)=0$ in Definition 8). Any $\ell_{x}(\cdot)$ satisfying (11) may be used in this case.

The next case of interest is (constrained) linear systems $x^{+}=$ $A x+G w, y=C x+v$ with quadratic penalties $\ell_{x}(x)=(1 / 2)|x|_{P_{0}}^{2}$ and $\ell_{i}(w, v)=(1 / 2)\left(|w|_{Q}^{2}+|v|_{R}^{2}\right)$ for penalty matrices $P_{0}, Q, R>$ 0 . We have the following result.

Proposition 15 (Constrained linear systems with quadratic costs). Assumption 11 is satisfied for $L Q$ estimation of a detectable system. In this case there exists $c>0$ and $\lambda<1$ such that for all $k \geq 1$

$$
\alpha\left(3 \underline{\gamma}_{x}^{-1}\left(3 k \bar{\gamma}_{w}(\|\mathbf{w}\|)\right), k\right) \leq c\|\mathbf{w}\| \lambda^{k}
$$

and the right-hand side is an exponential $\mathcal{K} \mathcal{L}$ function.
Proof: For a detectable linear system it can be shown that the $\mathcal{K} \mathcal{L}$ function $\alpha(\cdot)$ in Definition 8 satisfies an exponential decay rate

$$
\alpha(r, k) \leq c_{\alpha} r \bar{\lambda}^{k}, \quad 0<\bar{\lambda}<1
$$

The stage cost bounds satisfy

$$
\begin{aligned}
\underline{\gamma}_{x}(|x|) & =\underline{a}_{x}|x|^{2} & \bar{\gamma}_{x}(|x|) & =\bar{a}_{x}|x|^{2} \\
\underline{\gamma}_{w}(|w|) & =\underline{a}_{w}|w|^{2} & \bar{\gamma}_{w}(|w|) & =\bar{a}_{w}|w|^{2} \\
\underline{\gamma}_{v}(|v|) & =\underline{a}_{v}|v|^{2} & \bar{\gamma}_{v}(|v|) & =\bar{a}_{v}|v|^{2}
\end{aligned}
$$

in which $\underline{a}_{x}, \underline{a}_{w}$, and $\underline{a}_{v}\left(\bar{a}_{x}, \bar{a}_{w}\right.$, and $\left.\bar{a}_{v}\right)$ denote $(1 / 2)$ times the smallest (largest) singular values of $P_{0}, Q$, and $R$, respectively. Using these results, $\gamma_{x}^{-1}(\cdot)=\sqrt{\left(1 / \underline{a}_{x}\right)(\cdot)}$ and we have that

$$
3 \underline{\gamma}_{x}^{-1}\left(3 k \bar{\gamma}_{w}(\|\mathbf{w}\|)\right) \leq c_{\gamma} \sqrt{k}\|\mathbf{w}\|
$$

with $c_{\gamma}=3 \sqrt{3 k \bar{a}_{w} / \underline{a}_{x}}$. Applying $\alpha(\cdot)$ gives

$$
\alpha\left(3 \underline{\gamma}_{x}^{-1}\left(3 k \bar{\gamma}_{w}(\|\mathbf{w}\|)\right), k\right) \leq c_{\alpha} c_{\gamma}\|\mathbf{w}\| \sqrt{k} \bar{\lambda}^{k}
$$

We can then increase $\bar{\lambda}$ slightly and obtain an exponential bound, i.e., there exits $\lambda$ such that

$$
c_{\alpha} c_{\gamma}\|\mathbf{w}\| \sqrt{k} \bar{\lambda}^{k} \leq c\|\mathbf{w}\| \lambda^{k}, \quad \bar{\lambda}<\lambda<1
$$

and the result is established.

Note that for the LQ case, both Assumptions 11 and 12 are satisfied. Assumption 11 holds for any $a>0$ since $\lambda^{k}$ decays faster than $k^{-a}$. The LQ values of $p=1$ and $q=2$ satisfies $q>p / a$ in Assumption 12 for $a>1 / 2$.

\section{B. Implementation of the MIX and MAX estimators}

In order to computationally solve the MIX estimator (7), we can redefine it as

$$
\begin{aligned}
\min _{\chi(0), \boldsymbol{\omega}, \ell_{\max }} V_{T}(\chi(0), \boldsymbol{\omega}) & =(1+\delta) \frac{1}{T} \ell_{x}\left(\chi(0)-\bar{x}_{0}\right) \\
& +\frac{1}{T} \sum_{j=0}^{T-1} \ell_{i}(\omega(i), \nu(i))+\delta \ell_{\max }
\end{aligned}
$$

subject to (4) and

$$
-\ell_{\max }+\ell_{i}(\omega(i), \nu(i)) \leq 0, \quad 0 \leq i \leq T-1
$$

Then it has been transformed into a smooth optimization problem that can be solved by standard nonlinear optimization tools. A similar transformation can be applied on the MAX estimator. From this form of the definition, we can see the MIX and MAX estimators increase the dimension of the constraints by $T$, so they are usually slower and more difficult to solve than the SUM estimator, especially when $T$ is large. An MHE version of these estimators would significantly reduce the computational requirements.

\section{Convergent disturbances}

We are also interested in the convergence property of the state estimate when the disturbances are not only bounded but also converge to zero as time tends to infinity.

Remark 16. Notice that if the disturbances satisfy $|w(k)|,|v(k)| \rightarrow 0$ as $k \rightarrow \infty$, Definition 9 does not imply $\left|x\left(k ; x_{0}, \mathbf{w}\right)-x(k ; \hat{x}(0 \mid k), \widehat{\mathbf{w}})\right| \rightarrow 0$, because $\|\mathbf{w}\|_{0: k-1},\|\mathbf{v}\|_{0: k-1}$ (and $\|\widehat{\mathbf{w}}\|_{0: k-1},\|\widehat{\mathbf{v}}\|_{0: k-1}$ ) can remain large for large $k$ and corresponding small $|w(k)|$ and $|v(k)|$.

The convergence of the SUM estimator has already been established [1, Proposition 11], but the argument used in that case does 
not extend to the MIX and MAX estimators. Therefore a proof of the convergence of MIX and MAX estimators is a valuable topic for future research. See [3] for further thoughts on this issue.

When $\delta$ is taken small, the MIX estimator can be viewed as the SUM estimator with a small perturbation. Therefore, it is reasonable to conjecture that the MIX estimator is convergent for $\delta$ small enough. Obviously when $\delta$ is large this assumption does not hold and the convergence is not expected to hold. On the other hand, if $\delta$ is chosen too small, according to (20) and (21), a large RGAS upper bound could result. Therefore there is a trade off in the value of $\delta$ in order to ensure both good properties and good performance.

\section{EXAMPLE}

One physical nonlinear example is designed to illustrate the behaviors of the presented estimators, with random bounded noises for process and measurement disturbances. The simulation was performed with GNU Octave [8], and the software is available upon request.

Here we use the gas-phase irreversible reaction example proposed in [9]: $2 A \longrightarrow B$ with the reaction rate $r=k c_{A}^{2}, k=0.16$. We define the two states $x_{1}, x_{2}$ as the partial pressures of species $\mathrm{A}$ and $\mathrm{B}$, and the measurement as the total pressure. Assuming the ideal gas law holds and the batch reactor is well-mixed and isothermal, the model of system is $f(x)=\left[\begin{array}{c}-2 k x_{1}^{2} \\ k x_{1}^{2}\end{array}\right], x^{+}=\int_{0}^{\Delta} f(x) d t+w$ and $y=\left[\begin{array}{ll}1 & 1\end{array}\right] x+v$ with the sample time $\Delta=0.1$, and the initial state $x(0)=\left[\begin{array}{ll}3 & 1\end{array}\right]^{T}$. In the simulation we assume the plant suffers random noises $w \sim \mathcal{N}\left(0, Q_{w}\right)$ and $v \sim \mathcal{N}\left(0, R_{v}\right)$ where $Q_{w}=\operatorname{diag}\left(0.001^{2}, 0.001^{2}\right)$ and $R_{v}=0.1^{2}$. However, to ensure boundedness, hard constraints are included such that $\left|w_{1}\right|,\left|w_{2}\right| \leq$ $0.01,|v| \leq 1.0$. As in [9], the extended Kalman filter (EKF) given a poor initial guess $\bar{x}_{0}=\left[\begin{array}{ll}0.1 & 4.5\end{array}\right]^{T}, \Pi_{0}=\operatorname{diag}\left(6^{2}, 6^{2}\right)$ and accurate $Q_{w}, R_{v}$ covariance values is not stable. For comparison we also simulate the full information estimators with the same $\bar{x}_{0}$ and stage costs defined by $\ell_{x}(\chi):=\chi^{T} P_{0} \chi, P_{0}=\Pi_{0}^{-1}$ and $\ell_{i}(\omega, \nu):=\omega^{T} Q \omega+\nu^{T} R \nu, Q=Q_{w}^{-1}, R=R_{v}^{-1}$. The optimization is conducted over $T=11$ steps. The MIX estimator uses $\delta=1$. To provide sufficient statistical samples, a total of $s=300$ simulations are performed for each estimator.

Using the state estimate error defined in (10), Figure 1 shows the sample means (over all the runs) and standard deviations of estimate error $\tilde{x}_{1}(t)$ and $\tilde{x}_{2}(t)$ versus time of all four estimators. The EKF's estimate error does not converge to zero as time increases, and the estimator errors of MAX, MIX and SUM do converge to zero. To better compare their performances, we define the benchmark as $\left|\tilde{x}^{j}(T)\right|^{2}$ where $j$ denotes the $j$ th simulation run. The histogram at the final time is shown in Figure 2, which clearly indicates the performance differences. To make the comparison more straightforward, we can also look at the statistical expectation $\mathrm{E}\left(|\tilde{x}(T)|^{2}\right)$. In practice, the sample averages can be used to approximate the expectation

$$
\left\langle|\tilde{x}(T)|^{2}\right\rangle:=\frac{1}{s} \sum_{j=1}^{s}\left|\tilde{x}^{j}(T)\right|^{2}
$$

These values are given in the caption of Figure 2.

Similarly, we can use the achieved cost function values $V_{T}, V_{T}^{\max }$ and $V_{T}^{\text {sum }}$ as other performance benchmarks. The histograms and corresponding sample averages of $V_{T}$ are shown in Figure 3. Histograms of $V_{T}^{\max }$ and $V_{T}^{\text {sum }}$ are given in [10]. Not too surprisingly, each of the three estimators performs best on the benchmark corresponding to its own objective function. The 'Actual' plot in Figure 3 shows the value of the benchmark using the actual disturbances $(w, v)$ in the plant $\left(V_{T}(x(0), \mathbf{w})\right)$.
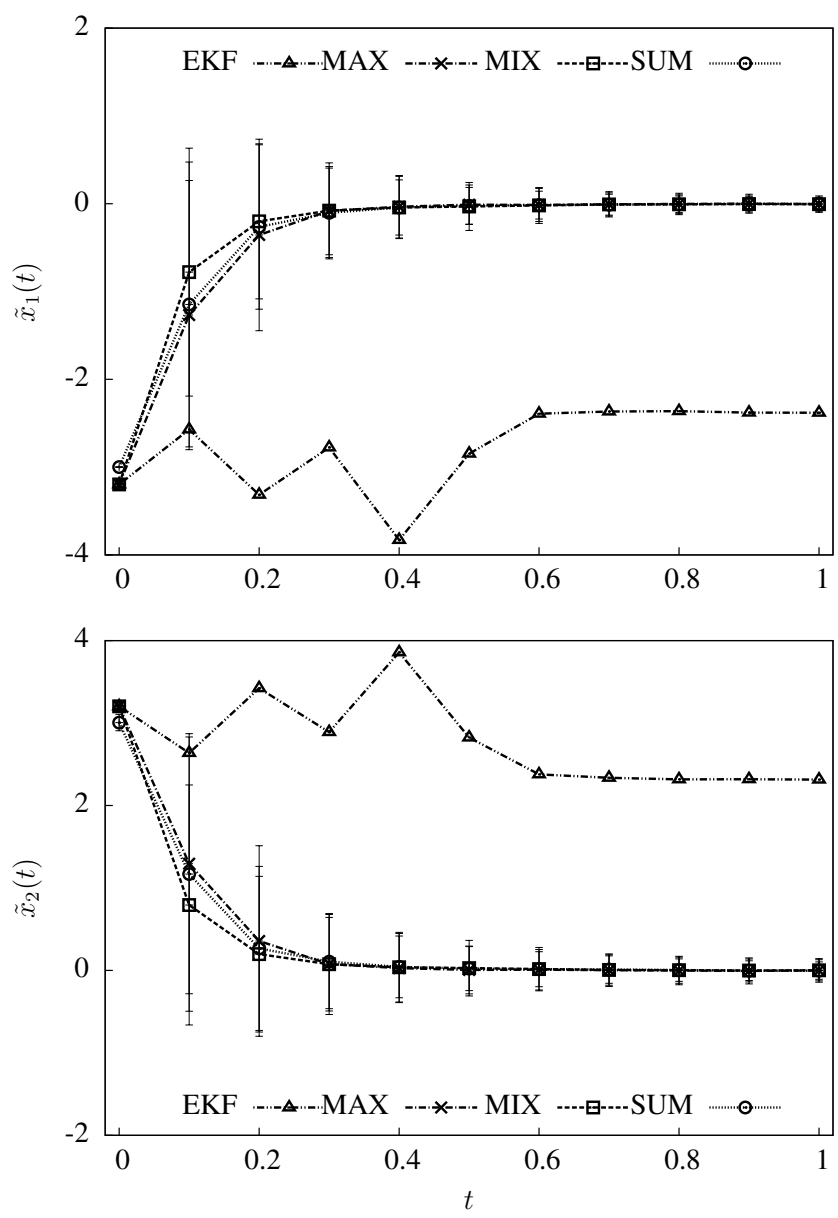

Fig. 1. Mean and standard deviation of estimate error $\tilde{x}(t)$ versus $t$ for MIX, MAX, and SUM full information estimators. The mean (only) of the EKF is shown for comparison.

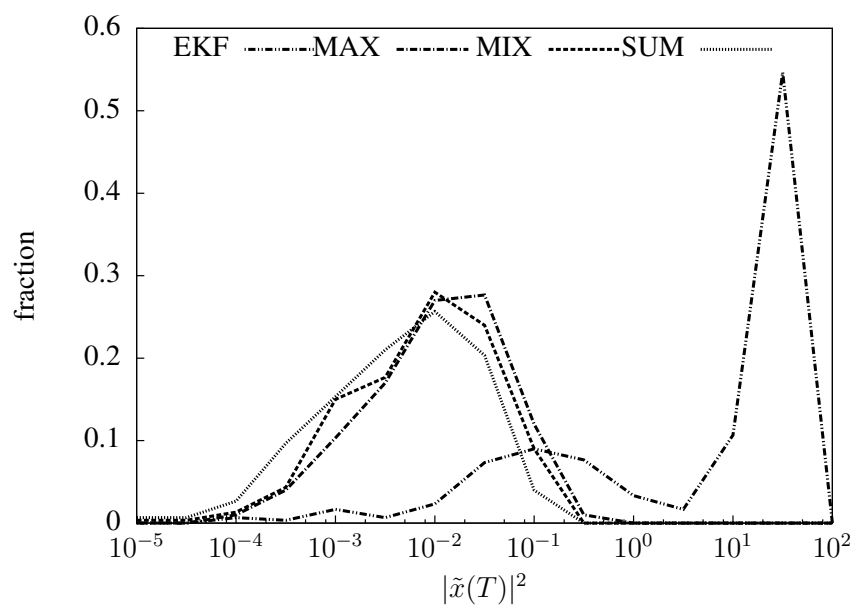

Fig. 2. Histogram of size of estimate error $|\tilde{x}(T)|^{2}$; EKF: $\left\langle|\tilde{x}(T)|^{2}\right\rangle=20.1$, MAX: $\left\langle|\tilde{x}(T)|^{2}\right\rangle=0.029$, MIX: $\left\langle|\tilde{x}(T)|^{2}\right\rangle=0.023$, SUM: $\left\langle|\tilde{x}(T)|^{2}\right\rangle=$ 0.015 


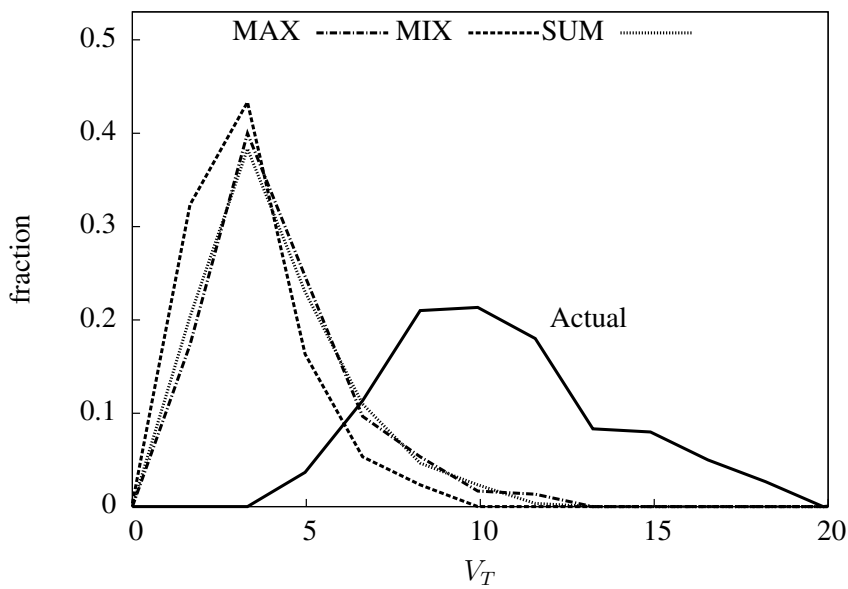

Fig. 3. Histogram of estimator mix cost $V_{T}$ for different estimators; MAX: $\left\langle V_{T}\right\rangle=4.2$, MIX: $\left\langle V_{T}\right\rangle=3.3$, SUM: $\left\langle V_{T}\right\rangle=4.1$, Actual: $\left\langle V_{T}\right\rangle=10.6$. For comparison "Actual" is the value of $V_{T}$ using the true rather than the optimally estimated disturbances.

\section{CONCLUSion}

Establishing robust global asymptotic stability (RGAS) ensures that the estimate error of a nonlinear estimator has an upper-bound depending on the sizes of the initial estimate error and the process and measurement disturbances. In previous work, RGAS of the full information estimator has been established for i-IOSS (detectable) nonlinear systems for convergent disturbances, i.e., disturbances that converge to zero as time increases to infinity. In applications, however, it is more reasonable to assume that the system disturbances are only bounded and not convergent. In this paper we defined a new form of the full information estimator that provides RGAS for detectable nonlinear systems for bounded disturbances. The new objective function includes the maximum over all stage costs as well as the standard sum of stage costs. The estimator is still optimization based and can incorporate constraints. To establish RGAS, we made one additional assumption relating the stage cost functions to the i-IOSS property. For observably nonlinear systems and constrained linear quadratic estimation of detectable systems, this assumption is always satisfied. A reformulation of this estimator is provided in order to implement the state estimator with standard nonlinear programming solvers.

We should emphasize that this RGAS definition does not automatically imply that the estimate error converges to zero for convergent disturbances due to the addition of the maximal stage cost term. It remains an open problem to establish that a single full information estimator has both RGAS and convergence properties. In future studies a moving horizon version of this new full information estimator will be valuable since it offers similar stability properties with a significantly smaller and tractable computational complexity.

\section{REFERENCES}

[1] J. B. Rawlings and L. Ji, "Optimization-based state estimation: Current status and some new results," J. Proc. Cont., vol. 22, pp. 1439-1444, 2012.

[2] J. Liu, "Moving horizon state estimation for nonlinear systems with bounded uncertainties," Chem. Eng. Sci., vol. 93, pp. 376-386, February 2013.

[3] W. Hu and L. Xie, "Optimization-based state estimation under bounded disturbances," Available: http://arxiv.org/abs/1410.5033, 2014.

[4] H. K. Khalil, Nonlinear Systems, 3rd ed. Upper Saddle River, NJ: Prentice-Hall, 2002.
[5] J. B. Rawlings and D. Q. Mayne, Model Predictive Control: Theory and Design. Madison, WI: Nob Hill Publishing, 2009, 576 pages, ISBN 978-0-9759377-0-9.

[6] E. D. Sontag and Y. Wang, "Output-to-state stability and detectability of nonlinear systems," Sys. Cont. Let., vol. 29, pp. 279-290, 1997.

[7] _ - "On the characterization of the input to state stability property," Sys. Cont. Let., vol. 24, pp. 351-359, 1995.

[8] J. W. Eaton, D. Bateman, and S. Hauberg, GNU Octave Manual Version 3. Network Theory Limited, 2008.

[9] E. L. Haseltine and J. B. Rawlings, "A critical evaluation of extended Kalman filtering and moving horizon estimation," TWMCC, Department of Chemical Engineering, University of Wisconsin-Madison, Tech. Rep. 2002-03, August 2002.

[10] L. Ji, J. B. Rawlings, W. Hu, A. Wynn, and M. Diehl, "Robust stability of moving horizon estimation under bounded disturbances," TWCCC, Department of Chemical and Biological Engineering, University of Wisconsin-Madison, Tech. Rep. 2013-01, November 2013. [Online]. Available: http://jbrwww.che.wisc.edu/tech-reports/twccc-2013-01.pdf 